\title{
A PRIME-DIVISOR FUNCTION
}

\section{J. KNOPFMACHER}

AbSTRaCt. This note studies the asymptotic mean values over arithmetical progressions, the general distribution of values, and the maximum order of magnitude, of a certain natural prime-divisor function of positive integers.

Consider the multiplicative arithmetical function $\beta$ defined by $\beta(1)=1$ and $\beta(n)=\alpha_{1} \alpha_{2} \cdots \alpha_{r}$ if $n=p_{1}^{\alpha_{1}} p_{2}^{\alpha_{2}} \cdots p_{r}^{\alpha_{r}}\left(p_{i}\right.$ prime, $\left.\alpha_{i}>0\right)$. Kendall and Rankin [2, p. 199] pointed out that this function has the finite mean value

$$
\lim _{N \rightarrow \infty} \frac{1}{N} \sum_{n=1}^{N} \beta(n)=\frac{\zeta(2) \zeta(3)}{\zeta(6)}=1.943 \cdots
$$

Strangely, perhaps, there appears to be virtually no other information available about this natural arithmetical function. (See note added in proof.) This note makes a more detailed study of its asymptotic properties.

\section{Average values and distribution.}

THEOREM 1. Let $r$ and $q$ denote relatively prime positive integers. Then.

$$
\begin{aligned}
\sum_{n \leqq x ; n \equiv r(\bmod q)} \beta(n)=\frac{1}{q}\left\{\frac{L\left(2, \chi_{0}\right) L\left(3, \chi_{0}\right)}{L\left(6, \chi_{0}\right)} x+\frac{L\left(\frac{1}{2}, \chi_{0}\right) L\left(\frac{3}{2}, \chi_{0}\right)}{L\left(3, \chi_{0}\right)} x^{1 / 2}\right. \\
\quad+\frac{L\left(\frac{1}{3}, \chi_{0}\right) L\left(\frac{2}{3}, \chi_{0}\right)}{L\left(2, \chi_{0}\right)} x^{1 / 3}+\frac{\bar{\chi}_{1}(r) L\left(\frac{1}{2}, \chi_{1}\right) L\left(\frac{3}{2}, \chi_{1}\right)}{L\left(3, \chi_{0}\right)} x^{1 / 2} \\
\left.+\sum_{\chi_{2}} \frac{\bar{\chi}_{2}(r) L\left(\frac{1}{3}, \chi_{2}\right) L\left(\frac{2}{3}, \chi_{2}^{2}\right)}{L\left(2, \chi_{0}\right)} x^{1 / 3}\right\} \\
+O\left(x^{3 / 10} \log ^{9 / 10} x \phi(q) q^{8 / 5}\right),
\end{aligned}
$$

where $\chi_{0}$ denotes the principal character $\bmod q$, and the terms in $\chi_{1}, \chi_{2}$ occur if and only if there exist characters $\chi_{1} \neq \chi_{0}, \chi_{2} \neq \chi_{0} \bmod q$ such that $\chi_{1}^{2}=\chi_{0}, \chi_{2}^{3}=\chi_{0}$.

Received by the editors November $21,1972$.

AMS (MOS) subject classifications (1970). Primary 10H25.

Key words and phrases. Asymptotic mean value, arithmetical progression, asymptotic distribution function, frequency, maximum order of magnitude.

(c) American Mathematical Society 1973 
Proof. Given any character $\chi \bmod q$, the Euler product formula for $L(z, \chi)$ leads to the equation

$$
\sum_{n=1}^{\infty} \chi(n) \beta(n) n^{-z}=\frac{L(z, \chi) L\left(2 z, \chi^{2}\right) L\left(3 z, \chi^{3}\right)}{L\left(6 z, \chi^{6}\right)} \quad[\operatorname{Re} z>1] .
$$

Therefore, if $L(z, \chi) L\left(2 z, \chi^{2}\right) L\left(3 z, \chi^{3}\right)=\sum_{n=1}^{\infty} c(n, \chi) n^{-z}[\operatorname{Re} z>1]$, then

$$
\sum_{n \leqq x} \chi(n) \beta(n)=\sum_{k m^{6} \leqq x} c(k, \chi) \chi^{6}(m) \mu(m)=\sum_{m \leqq x^{1 / 6}} \chi^{6}(m) \mu(m) \sum_{k \leqq x / m^{6}} c(k, \chi) .
$$

Now, by equations (68-71) of Richert [7], we have:

$$
\begin{aligned}
\sum_{n \leqq x} c\left(n, \chi_{0}\right)= & \frac{\phi(q)}{q}\left\{x L\left(2, \chi_{0}\right) L\left(3, \chi_{0}\right)+x^{1 / 2} L\left(\frac{1}{2}, \chi_{0}\right) L\left(\frac{3}{2}, \chi_{0}\right)\right. \\
& \left.+O\left(x^{3 / 10} \log ^{9 / 10} x \phi(q) q^{8 / 5}\right) ; \quad+x^{1 / 3} L\left(\frac{1}{3}, \chi_{0}\right) L\left(\frac{2}{3}, \chi_{0}\right)\right\}
\end{aligned}
$$

(ii) $\sum_{n \leqq x} c\left(n, \chi_{1}\right)=\frac{\phi(q)}{q} x^{1 / 2} L\left(\frac{1}{2}, \chi_{1}\right) L\left(\frac{3}{2}, \chi_{1}\right)+O\left(x^{3 / 10} \log ^{9 / 10} x \phi(q) q^{4 / 3}\right)$

$$
\text { if } \chi_{1}^{2}=\chi_{0} \text { but } \chi_{1} \neq \chi_{0} \text {; }
$$

$$
\begin{array}{r}
\sum_{n \leqq x} c\left(n, \chi_{2}\right)=\frac{\phi(q)}{q} x^{1 / 3} L\left(\frac{1}{3}, \chi_{2}\right) L\left(\frac{2}{3}, \chi_{2}^{2}\right)+O\left(x^{3 / 10} \log ^{9 / 10} x \phi(q) q^{3 / 5}\right) \\
\text { if } \chi_{2}^{3}=\chi_{0} \text { but } \chi_{2} \neq \chi_{0} ;
\end{array}
$$

(iv) $\sum_{n \leqq x} c(n, \chi)=O\left(x^{3 / 10} \log ^{9 / 10} x q^{8 / 5}\right)$ for all other $\chi$.

It follows for example that

$$
\begin{aligned}
\sum_{n \leqq x} \chi_{1}(n) \beta(n)= & \sum_{m \leqq x^{1 / 6}} \chi_{1}^{6}(m) \mu(m)\left\{\frac{\phi(q) x^{1 / 2}}{q m^{3}} L\left(\frac{1}{2}, \chi_{1}\right) L\left(\frac{3}{2}, \chi_{1}\right)\right. \\
& \left.\quad+O\left(\left(x / m^{6}\right)^{3 / 10} \log ^{9 / 10}\left(x / m^{6}\right) \phi(q) q^{4 / 3}\right)\right\} \\
= & \frac{\phi(q)}{q} x^{1 / 2} L\left(\frac{1}{2}, \chi_{1}\right) L\left(\frac{3}{2}, \chi_{1}\right)\left[\frac{1}{L\left(3, \chi_{0}\right)}+O\left(x^{-1 / 3}\right)\right] \\
& +O\left(x^{3 / 10} \log ^{9 / 10} x \phi(q) q^{4 / 3}\right) \\
= & \frac{\phi(q)}{q} x^{1 / 2} \frac{L\left(\frac{1}{2}, \chi_{1}\right) L\left(\frac{3}{2}, \chi_{1}\right)}{L\left(3, \chi_{0}\right)}+O\left(x^{3 / 10} \log ^{9 / 10} x \phi(q) q^{4 / 3}\right) .
\end{aligned}
$$

Similarly one can determine estimates for $\sum_{n \leqq x} \chi(n) \beta(n)$ in the other cases. The theorem then follows from the equation

$$
\sum_{n \leqq x ; n \equiv r(\bmod q)} \beta(n)=\sum_{n \leqq x} \beta(n) \frac{1}{\phi(q)} \sum_{\chi \bmod q} \chi(n) \bar{\chi}(r) .
$$


Corollary.

$$
\sum_{n \leqq x} \beta(n)=\frac{\zeta(2) \zeta(3)}{\zeta(6)} x+\frac{\zeta\left(\frac{1}{2}\right) \zeta\left(\frac{3}{2}\right)}{\zeta(3)} x^{1 / 2}+\frac{\zeta\left(\frac{1}{3}\right) \zeta\left(\frac{2}{3}\right)}{\zeta(2)} x^{1 / 3}+O\left(x^{3 / 10} \log ^{9 / 10} x\right) .
$$

REMARK. By using a theorem of Schmidt [5] concerning $\sum_{n \leqq x} c(n)$ where $\sum c(n) n^{-z}=\zeta(z) \zeta(2 z) \zeta(3 z)[\operatorname{Re} z>1]$, one can sharpen the error term in this corollary to $O\left(x^{7 / 27} \log ^{2} x\right)$. For a more general, though less sharp, version of the corollary, see the author [3]. (See note added in proof.)

Next, by applying a theorem of Schoenberg [6, p. 319], one obtains

THEOREM 2. The function $\beta$ possesses an asymptotic distribution function

$$
F(x)=\lim _{N \rightarrow \infty} \frac{1}{N} \operatorname{card}\{n \leqq N: \beta(n) \leqq x\} .
$$

This function is discrete, and the characteristic function of $F\left(e^{x}\right)$ is

$$
\int_{-\infty}^{\infty} e^{i t x} d F\left(e^{x}\right)=\prod_{\text {primes } p}\left(1-p^{-1}\right)\left\{1+\sum_{r=1}^{\infty} p^{-r} e^{i t \log r}\right\} .
$$

Remark. As part of a different discussion, J. Ridley and the author [4] have shown that, for each $k=1,2, \cdots$, the function $\beta$ has a finite $k$ th moment

$$
\lim _{N \rightarrow \infty} \frac{1}{N} \sum_{n=1}^{N}[\beta(n)]^{k}=\prod_{\text {primes } p}\left\{1+\sum_{r=2}^{\infty}\left[r^{k}-(r-1)^{k}\right] p^{-r}\right\} .
$$

It may also be mentioned that a slight modification of a technique of Kendall and Rankin [2, p. 204] (who are concerned with the total number $a(n)$ of nonisomorphic abelian groups of order $n$ ) leads to an explicit formula for the frequency

$$
F_{m}=\lim _{N \rightarrow \infty} \frac{1}{N} \operatorname{card}\{n \leqq N: \beta(n)=m\} .
$$

Since this formula is relatively involved, we do not give it in detail, but we note that by combining certain of the frequencies $P_{m}$ calculated for $a(n)$ in [2, p. 205] one obtains:

$$
\begin{array}{ll}
F_{1}=P_{1}=6 / \pi^{2}=0.6079 \cdots, & F_{2}=P_{2}=0.2008 \cdots, \\
F_{3}=P_{3}=0.0742 \cdots, & F_{4}=P_{4}+P_{5}=0.0542 \cdots, \\
F_{5}=P_{7}=0.0147 \cdots, & F_{6}=P_{6}+P_{11}=0.0215 \cdots .
\end{array}
$$

Thus $F_{1}+\cdots+F_{6}=0.9733 \cdots$, which emphasizes how closely the values of $\beta$ cluster about its mean value $1.943 \cdots$. In fact, after a computer 
check on the "empirical" frequencies of $\beta$ over the range $1 \leqq n \leqq 10,000$, $\mathrm{J}$. Ridley has very kindly provided the following figures for the actual frequencies in this range: $F_{1}^{\prime}=0.6083, F_{2}^{\prime}=0.2008, F_{3}^{\prime}=0.0744, F_{4}^{\prime}=0.0541$, $F_{5}^{\prime}=0.0151, F_{6}^{\prime}=0.0216$. Here $F_{1}^{\prime}+\cdots+F_{6}^{\prime}=0.9743$.

\section{Maximum order of magnitude.}

THEOREM 3. Given any $\varepsilon>0$ there exists an integer $n_{0}(\varepsilon)$ such that

while

$$
\beta(n)<3^{(1 / 3)(1+\varepsilon) \log n / \log \log n} \text { for all } n \geqq n_{0}(\varepsilon),
$$

$$
\beta(n)>3^{(1 / 3)(1-\varepsilon) \log n / \log \log n} \text { for infinitely many } n .
$$

Proof. The argument is parallel to one whereby Hardy and Wright [1] proved an analogous theorem for the divisor function $d(n)$. Firstly, one notes by induction that $a \leqq 3^{a / 3}$ for $a=1,2, \cdots$. Hence, for $p \geqq 3^{1 / 3 \delta}$ $(\delta>0)$ and $a=1,2, \cdots$,

$$
\frac{a}{p^{a \delta}} \leqq \frac{a}{3^{a / 3}} \leqq 1 .
$$

If $n=p_{1}^{a_{1}} p_{2}^{a_{2}} \cdots p_{r}^{a_{r}}\left(p_{i}\right.$ prime, $\left.a_{i}>0\right)$, it follows that

$$
\frac{\beta(n)}{n^{\delta}}=\prod_{1}^{r} \frac{a_{i}}{p_{i}^{a_{i} \delta}} \leqq \prod_{\text {primes }} \prod_{p \leqq 3^{1 / 3 \delta}}(\delta \log 2)^{-1}<\exp \left(\frac{3^{1 / 3 \delta}}{\delta \log 2}\right) .
$$

If $\delta=\left(1+\frac{1}{2} \varepsilon\right) \log 3 / 3 \log \log n(\varepsilon>0)$, then

$$
\frac{3^{1 / 3 \delta}}{\delta \log 2}=\frac{(\log n)^{1 /(1+\varepsilon / 2)} \log \log n}{\left(1+\frac{1}{2} \varepsilon\right) \log 2 \cdot \log 3^{1 / 3}}<\frac{\varepsilon \log 3^{1 / 3} \cdot \log n}{2 \log \log n}
$$

for $n$ sufficiently large. Hence the upper inequality follows.

For the lower inequality, let $N=\left(p_{1} p_{2} \cdots p_{r}\right)^{3}$ where $p_{1}<\cdots<p_{r}$ denote the first $r$ primes. Then

$$
\log \beta(N)=r \log 3=\pi\left(p_{r}\right) \log 3 \geqq \frac{\log \left(p_{1} p_{2} \cdots p_{r}\right) \cdot \log 3}{\log p_{r}},
$$

in a similar way to the situation in $[1$, p. 263]. Hence, in the same way, there is a constant $C$ such that

$$
\log \beta(N)>\frac{\log N \cdot \log 3^{1 / 3}}{\log \log N+C}>\frac{(1-\varepsilon) \log 3^{1 / 3} \cdot \log N}{\log \log N}
$$

for $N$ sufficiently large, i.e. for $r$ sufficiently large.

THEOREM 4. Given any $\varepsilon>0, \beta(n)<3^{(1 / 3)(1+\varepsilon) \log \log n}$ for "almost all" $n$, i.e. all $n$ outside some set of asymptotic density zero. 
Proof. The inequality $a \leqq 3^{a / 3}(a=1,2, \cdots)$ implies that $\beta(n) \leqq 3^{\Omega(n) / 3}$ where $\Omega(n)$ is the sum of the exponents of the prime divisors of $n$. The theorem then follows from Theorem 431 of [1], which states that $\Omega(n)$ has "normal order" $\log \log n$.

REMARK. One cannot expect a similar lower inequality, since for example $\beta$ takes the value 1 on all square-free integers, and these have positive density $6 / \pi^{2}$.

NOTE ADDED IN PROOF. In a recent paper, The number of square-full divisors of an integer, Proc. Amer. Math. Soc. 34 (1972), 79-80, D. Suryanarayana and R. Sita Rama Chandra Rao established the above corollary to Theorem 1, with an error estimate slightly weaker than that obtainable with the aid of Schmidt's theorem [5].

\section{REFERENCES}

1. G. H. Hardy and E. M. Wright, An introduction to the theory of numbers, 3rd ed., Clarendon Press, Oxford, 1954. MR 16, 673.

2. D. G. Kendall and R. A. Rankin, On the number of abelian groups of a given order, Quart. J. Math. Oxford Ser. 18 (1947), 197-208. MR 9, 226.

3. J. Knopfmacher, Arithmetical properties of finite rings and algebras, and analytic number theory. II, J. Reine Angew. Math. 254 (1972), 74-99.

4. J. Knopfmacher and J. Ridley, Prime-independent arithmetical functions, Ann. Mat. Pura Appl. (to appear).

5. P. G. Schmidt, Zur Anzahl Abelscher Gruppen gegebener Ordnung. II, Acta Arith. 13 (1967/68), 405-417. MR 37 \#190.

6. I. J. Schoenberg, On asymptotic distributions of arithmetical functions, Trans. Amer. Math. Soc. 39 (1936), 315-330.

7. H. E. Richert, Über die Anzahl Abelscher Gruppen gegebener Ordnung. II, Math. Z. 58 (1953), 71-84. MR 14, 945.

Department of Mathematics, University of the WitwatersRand, JohanNesburg, SOUTH AFrica 